\title{
Microscopic Study of a Pressure-Induced Ferromagnetic-Spin-Glass Transition in the Geometrically Frustrated Pyrochlore $\left(\mathrm{Tb}_{1-x} \mathrm{La}_{x}\right)_{2} \mathrm{Mo}_{2} \mathrm{O}_{7}$
}

\author{
A. Apetrei ${ }^{1}{ }^{\text {I. Mirebeau },}{ }^{1}$ I. Goncharenko, ${ }^{1}$ D. Andreica, ${ }^{2,3}$ and P. Bonville ${ }^{4}$ \\ ${ }^{1}$ Laboratoire Léon Brillouin, CEA-CNRS, CE-Saclay, 91191 Gif-sur-Yvette, France \\ ${ }^{2}$ Laboratory for Muon-Spin Spectroscopy, Paul Scherrer Institut, 5232 Villigen-PSI, Switzerland \\ ${ }^{3}$ Faculty of Physics, Babes-Bolyai University, 400084 Cluj-Napoca, Romania \\ ${ }^{4}$ Service de Physique de l'Etat Condensé, CEA-CNRS, CE-Saclay, 91191 Gif-Sur-Yvette, France
}

(Received 24 April 2006; published 15 November 2006)

\begin{abstract}
We have studied $\left(\mathrm{Tb}_{1-x} \mathrm{La}_{x}\right)_{2} \mathrm{Mo}_{2} \mathrm{O}_{7}$ pyrochlores by neutron diffraction and $\mu \mathrm{SR}$ at ambient and under applied pressure. ( $\mathrm{Tb}, \mathrm{La}$ ) substitution expands the lattice and induces a change from a spin-glass-like state $(x=0)$ to a noncollinear ferromagnet $(x=0.2)$. In the ferromagnetic structure, the Tb moments orient close to their local anisotropy axes as for an ordered spin ice, while the Mo ones orient close to the net moment. The temperature dependence of the $\mu \mathrm{SR}$ relaxation rates and static local fields suggests a second transition of dynamical nature below the Curie transition. Under pressure, the long range order breaks down and a spin-glass-like state is recovered. The whole set of data provides a microscopic picture of the spin correlations and fluctuations in the region of the ferromagnetic-spin-glass threshold.
\end{abstract}

DOI: 10.1103/PhysRevLett.97.206401

PACS numbers: 71.30.+h, 71.27.+a, 75.25.+z

In the pyrochlore compounds $R_{2} M_{2} \mathrm{O}_{7}$, both the rare earth $R^{3+}$ and the $M^{4+}$ transition metal form a threedimensional network of corner-sharing tetrahedra. The pyrochlore lattice is geometrically frustrated for both antiferromagnetic (AF) and ferromagnetic (F) nearestneighbor exchanges, leading to intriguing magnetic states such as spin liquids, spin ices, or chemically ordered spin glasses $[1,2]$.

In $R_{2} \mathrm{Mo}_{2} \mathrm{O}_{7}$ pyrochlores, electrical and magnetic properties strongly depend on the rare earth ionic radius $r$. Compounds with a small ionic radius (Y, Dy, and $\mathrm{Tb}$ ) are spin-glass (SG) insulators, whereas those with $\mathrm{Gd}, \mathrm{Sm}$, and $\mathrm{Nd}$ are $\mathrm{F}$ metals. The $\left(R R^{\prime}\right)_{2} \mathrm{Mo}_{2} \mathrm{O}_{7}$ series shows a universal dependence $[3,4]$ of the transition temperature versus $r$, suggesting that the sign of Mo-Mo interactions controls the formation of the spin-glass-ferromagnetic state. Photoemission experiments and band structure calculations $[5,6]$ point out that the concomitant changes of the transport and magnetic properties come from strong electron correlations in the $\operatorname{Mo}\left(t_{2 g}\right)$ band near the Fermi level.

Up to now, there has been no microscopic investigation of the SG-F threshold, so as to follow the changes in the magnetic correlations and spin fluctuations. All microscopic studies deal with compounds far from the threshold radius $r_{c} . \mathrm{Y}_{2} \mathrm{Mo}_{2} \mathrm{O}_{7}$ and $\mathrm{Tb}_{2} \mathrm{Mo}_{2} \mathrm{O}_{7}$ with $r<r_{c}$ were intensively studied [7-10], since the $\mathrm{SG}$ transition is surprising with regards to their chemical order. $\mathrm{Nd}_{2} \mathrm{Mo}_{2} \mathrm{O}_{7}$ with $r>r_{c}$ showing a giant abnormal Hall effect was also much studied $[11,12]$. F compounds near the threshold ( $\mathrm{Sm}$ and $\mathrm{Gd}$ ) are difficult to study by neutrons due to their strong absorption. So the microscopic evolution of magnetism throughout the threshold was not clarified so far.

To understand the role of interatomic distances in the SG-F transition, the most direct way is to combine applied pressure and chemical pressure. In $\mathrm{Gd}_{2} \mathrm{Mo}_{2} \mathrm{O}_{7}$, magnetic measurements showed that an applied pressure as well as (Gd, Dy) substitution could suppress the ferromagnetic transition and induce spin-glass-like anomalies [13,14]. Here we have studied the $\left(\mathrm{Tb}_{1-x} \mathrm{La}_{x}\right)_{2} \mathrm{Mo}_{2} \mathrm{O}_{7}$ system by neutron diffraction and $\mu \mathrm{SR}$ at ambient and applied pressure. Starting from the spin glass $\mathrm{Tb}_{2} \mathrm{Mo}_{2} \mathrm{O}_{7}$, the dilution by a nonmagnetic $\mathrm{La}$ ion induces long range magnetic order. By neutron diffraction, we follow this evolution at a microscopic level. For $x=0.2$, we observe a new ordered magnetic structure, involving noncollinear ferromagnetic arrangement of both $\mathrm{Tb}$ and Mo moments. Under pressure, the $x=0.2$ sample transforms into a spin glass similar to $\mathrm{Tb}_{2} \mathrm{Mo}_{2} \mathrm{O}_{7}$. While neutron diffraction probes magnetic correlations, the $\mu \mathrm{SR}$ technique allows one to study spin fluctuations. For $x=0.2$, we observe by $\mu \mathrm{SR}$ a dynamical anomaly at a temperature $T^{\star}$ well below the Curie temperature $T_{C}$. Under pressure, the two temperatures seem to merge. The whole data provide the first and complete microscopic picture of the F-SG threshold.

The crystal structure of $\left(\mathrm{Tb}_{1-x} \mathrm{La}_{x}\right)_{2} \mathrm{Mo}_{2} \mathrm{O}_{7}$ powders $(x=0,0.05,0.1,0.15$, and 0.2) was characterized at $300 \mathrm{~K}$ by combining $\mathrm{x}$-ray and neutron diffraction. FULLPROF refinements [15] show that all samples crystallize in the $F d \overline{3} m$ space group, with a lattice constant $a$ between 10.3124(7) $(x=0)$ and 10.3787(8) $\AA(x=0.2)$. The $x=0.1$ sample $[a=10.3461(8) \AA]$ is above the critical threshold $\left(a_{c} \sim 10.33 \AA\right)$. The lattice constant was measured under pressure for $x=0$ and 0.2 on the ID27 beam line of the European Synchrotron Radiation Facility (ESRF). Susceptibility data show an evolution from a spin glass with irreversibilities below $T_{\mathrm{SG}} \sim 22 \mathrm{~K}(x=0)$ towards a ferromagnetic state with a strong increase of magnetization below $T_{C} \sim 58 \mathrm{~K}(x=0.2)$.

Magnetic diffraction patterns were recorded on the powder diffractometers G61 and G41 of the Laboratoire Léon 
Brillouin (LLB) at ambient pressure and under pressure on G61 in its high pressure version [16]. They show the evolution of the magnetic order when going through the critical threshold by substitution of Tb for La [Fig. 1(a)] and by applied pressure [Fig. 1(b)]. It is clear evidence that magnetic changes induced by chemical and applied pressures are microscopically equivalent.

In $\mathrm{Tb}_{2} \mathrm{Mo}_{2} \mathrm{O}_{7}(x=0)$, short range order yields diffuse maxima around $q=1$ and $2 \AA^{-1}$. An intense signal at low $q$ values reveals ferromagnetic correlations. For $x=0.1$, Lorentzian peaks start to grow at the position of the diffuse maxima, revealing the onset of mesoscopic magnetic order. For $x=0.2$, the low $q$ signal almost disappears, and we clearly see magnetic Bragg peaks showing long range magnetic order. When we apply pressure on the $x=0.2$ sample, the intensity of the Bragg peaks decreases, and the ferromagnetic correlations and diffuse maxima start to grow. The magnetic pattern for $x=0.2$ at $1.05 \mathrm{GPa}$ (3.7 $\mathrm{GPa})$ is very similar to that for $x=0.1(x=0)$ at ambient pressure.

In the range $q=0.5-2.5 \AA^{-1}$, we analyzed the magnetic correlations in $\mathrm{Tb}_{2} \mathrm{Mo}_{2} \mathrm{O}_{7}$ by a short range magnetic model as in Ref. [8]. A fit of the diffuse magnetic intensity by the sum of radial correlation functions yields the spin correlation parameters $\gamma$ up to the fourth coordination shell $(\sim 7.3 \AA)$. The $\mathrm{Tb}-\mathrm{Tb}$ correlations are $\mathrm{F}\left(\gamma_{1,3,4}>0\right)$, while the Tb-Mo are $\operatorname{AF}\left(\gamma_{2}<0\right)$ in agreement with previous results [8]. The AF Mo-Mo correlations yielding the frustration in the SG state are not seen, being about 50 times smaller than the $\mathrm{Tb}-\mathrm{Tb}$ ones due to the smaller Mo moment. The intense signal below $0.5 \AA^{-1}$ was not detected in previous patterns measured in a higher $q$ range $[8,9]$. It cannot be accounted for by the short range mag-

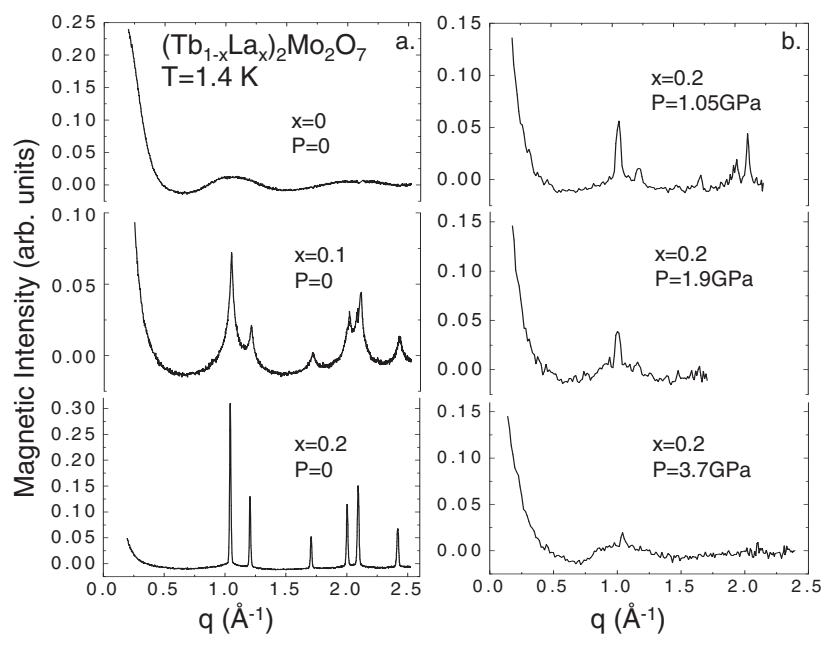

FIG. 1. Magnetic intensity of $\left(\mathrm{Tb}_{1-x} \mathrm{La}_{x}\right)_{2} \mathrm{Mo}_{2} \mathrm{O}_{7}$ at $1.4 \mathrm{~K}$ versus the scattering vector $q=4 \pi \sin \theta / \Lambda$. The neutron wavelength is $\Lambda=4.741 \AA$. A spectrum in the paramagnetic phase $(70 \mathrm{~K})$ was subtracted, and the magnetic intensity was scaled to the (222) nuclear peak intensity. netic model, even when increasing the correlation range up to the seventh coordination shell. We attribute it to $\mathrm{Tb}-\mathrm{Tb}$ ferromagnetic correlations and evaluate their length scale to $20(8) \AA$.

The magnetic Bragg peaks observed for $x=0.2$ belong to the face centered cubic lattice, showing that the magnetic structure is derived from the chemical one of $F d \overline{3} m$ symmetry by a propagation vector $\mathbf{k}=\mathbf{0}$. The two magnetic peaks (200) and (220), forbidden in the pyrochlore structure, suggest a noncollinear F structure.

The magnetic structure was solved by a systematic search, using the program BASIREPS [17] and symmetryrepresentation analysis [18] combined with FULLPROF [15]. Since neither a collinear $\mathrm{F}$ structure nor the $\mathbf{k}=\mathbf{0} \mathrm{AF}$ structure allowed by $F d \overline{3} m$ symmetry were compatible with the data, we searched for a solution in the space group $I_{41} /$ amd, the highest subgroup allowing $\mathrm{F}$ and $\mathrm{AF}$ components simultaneously. The best refinement $\left(R_{B}=4 \%\right)$ is shown in Fig. 2(a). In the ordered structure with $\mathbf{k}=\mathbf{0}$, the four tetrahedra of the unit cell are equivalent, for both $\mathrm{Tb}$ and Mo lattices. In a given $\mathrm{Tb}$ tetrahedron [inset in Fig. 2(a)], the $\mathrm{Tb}^{3+}$ moments orient in the local spin ice structure [1] with a small angle $\theta_{t}=11.6^{\circ}$ at $1.4 \mathrm{~K}$ with the local $\langle 111\rangle$ anisotropy axes connecting the center to the vertices. Their F component orders along a [001] axis. The Mo moments align close to a [001] axis [inset in Fig. 2(a)], with a slight tilting by the angle $\theta_{m}=6.8^{\circ}$ at $1.4 \mathrm{~K}$ towards the local $\langle 111\rangle$ axes. Both Mo-Mo and Tb-Mo correlations are $\mathrm{F}$, in contrast with the spin glass.

As a striking feature, long range order (LRO) is induced by diluting the $\mathrm{Tb}$ lattice with a nonmagnetic ion. It proves that the main effect of dilution is the lattice expansion. From the lattice constant, a small La content $(x \sim 0.06)$ should induce the SG-F transition. We notice that $\mathrm{Tb}$ magnetism should play a role in this transition together

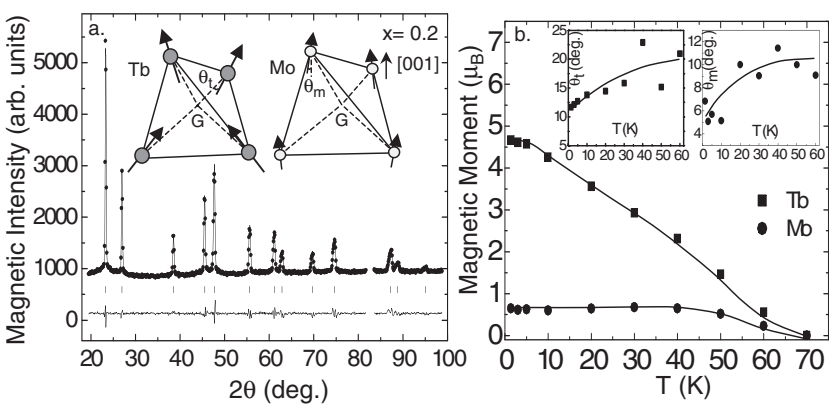

FIG. 2. (a) Magnetic intensity in the $x=0.2$ sample at $1.5 \mathrm{~K}$ versus the scattering angle $2 \theta$, with $\Lambda=2.426 \AA$. A spectrum at $70 \mathrm{~K}$ was subtracted. The solid lines show the best refinement $\left(R_{B}=4 \%\right)$ and the difference spectrum (bottom). In the inset is the magnetic structure of the $\mathrm{Tb}$ and Mo tetrahedra. (b) Ordered magnetic moments versus temperature. In the inset are the angles $\theta_{t}$ and $\theta_{m}$ made by $\mathrm{Tb}$ and Mo moments with the local anisotropy $\langle 111\rangle$ and the [001] axes, respectively. The solid lines are guides to the eye. 
with the change in sign of the Mo-Mo interaction, since $\left(\mathrm{Y}_{1-x} \mathrm{La}_{x}\right)_{2} \mathrm{Mo}_{2} \mathrm{O}_{7}$ compounds do not show LRO [19]. At higher dilution $(x=0.2)$, the Bragg peaks are better defined, but they still coexist with short range order (SRO), as shown by a diffuse scattering which increases below $40 \mathrm{~K}$. The SRO has the same symmetry as the LRO. It likely arises from the different local ( $\mathrm{Tb}, \mathrm{La}$ ) environments.

As for the LRO moments [Fig. 2(b)], $M_{\text {Mo }}$ is almost $T$-independent below $40 \mathrm{~K}$ while $M_{\mathrm{Tb}}$ keeps increasing below $T_{C}$. At $1.4 \mathrm{~K}$, we obtain $M_{\mathrm{Tb}}=4.66(2) \mu_{B}$ and $M_{\mathrm{Mo}}=0.64(3) \mu_{B}$. The SRO moments are correlated over about $20 \AA$ and evaluated to $4.3(2) \mu_{B}$ (Tb) and $0.2(5) \mu_{B}$ (Mo). The total moments of 6.3(1) $\mu_{B}$ (Tb) and $0.7(2) \mu_{B}$ (Mo) are still well reduced from the free ion values of $9 \mu_{B}$ and $2 \mu_{B}$, respectively. For Tb, this strong reduction could be explained mainly by crystal field effects. As for Mo, it could arise from orbital frustration or the proximity of the threshold leading to quantum fluctuations. The two tilting angles slightly decrease with decreasing temperature [inset in Fig. 2(b)].

The noncollinear structure for both $\mathrm{Tb}^{3+}$ and $\mathrm{Mo}^{4+}$ originates from the uniaxial anisotropy of the $\mathrm{Tb}^{3+}$ ion, which brings spin ice frustration to the ferromagnetic phase. The ground state is determined by Mo-Mo F exchange interactions like in $\mathrm{Nd}_{2} \mathrm{Mo}_{2} \mathrm{O}_{7}$ [11], while in the "ordered spin ice" $\mathrm{Tb}_{2} \mathrm{Sn}_{2} \mathrm{O}_{7}$ with similar orientation of $\mathrm{Tb}^{3+}$ moments [20] it results from $\mathrm{F}$ dipolar interactions between the $\mathrm{Tb}^{3+}$ ions.

Under pressure, the ordered moments $(x=0.2)$ decrease and reorient (Fig. 3). For $P=1.05 \mathrm{GPa}$, we observe the coexistence of LRO and SRO phases of the same symmetry. The LRO Tb moments keep the local spin ice configuration with a different $\theta^{\prime}{ }_{t}=28.3^{\circ}$. Mo moments also turn to a local spin ice order, making an angle $\theta^{\prime}{ }_{m}=$ $7.3^{\circ}$ with the local $\langle 111\rangle$ axes. The ordering temperature decreases with increasing pressure. At $3.7 \mathrm{GPa}$, the Bragg peaks disappear. From our fits, we obtain short range $\mathrm{F} \mathrm{Tb}$ $\mathrm{Tb}\left(\gamma_{1,3,4}>0\right)$ and AF Tb-Mo correlations $\left(\gamma_{2}<0\right)$, with a ferromagnetic correlation length of 18(7) $\AA$. These parameters are similar to those of $\mathrm{Tb}_{2} \mathrm{Mo}_{2} \mathrm{O}_{7}$. Our recent results also reveal the same behavior in $\mathrm{Gd}_{2} \mathrm{Mo}_{2} \mathrm{O}_{7}$ under pressure [21].

$\mu \mathrm{SR}$ measurements (Fig. 4) shed new light on the magnetic order by probing the spin fluctuations and the static local field below $T_{C}$. The recent availability of $\mu \mathrm{SR}$ under pressure allows us to probe them on both sides of the threshold. We measured the $x=0.2$ sample at ambient pressure on the GPS and GPD instruments of the Paul Scherrer Institut (PSI) and under a pressure of $1.3 \mathrm{GPa}$ on GPD.

Sample $\mu$ SR spectra above $T_{C}$ were best fitted with a stretched exponential function $P_{Z}(t)=\exp (-\lambda t)^{\beta}$. Below $T_{C}, \quad P_{Z}(t)$ was fitted by the function $P_{Z}(t)=$ $\left[\exp \left(-\lambda_{Z} t\right)^{\beta}+2 \exp \left(-\lambda_{T} t\right) \cos \left(\gamma_{\mu}\left\langle B_{\mathrm{loc}}\right\rangle t\right)\right] / 3$, expected for the magnetically ordered state of a powder sample
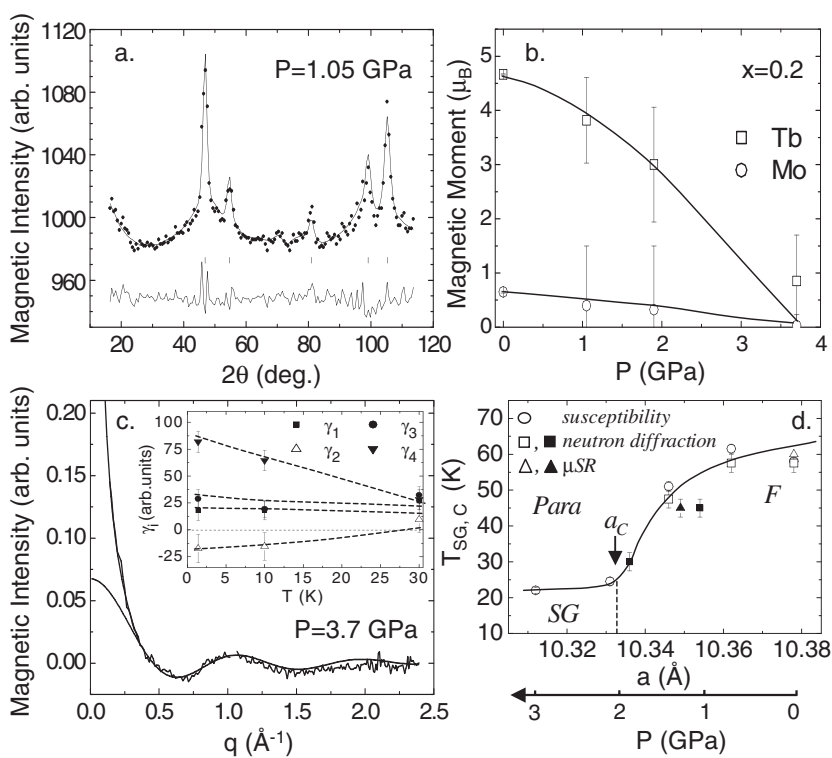

FIG. 3. (a) Magnetic intensity in $x=0.2$ sample at $1.4 \mathrm{~K}$ and $P=1.05 \mathrm{GPa}$, with $\Lambda=4.741 \AA$. The solid lines show the best refinement $\left(R_{B}=20 \%\right)$ and the difference spectrum; (b) magnetic moment versus pressure; (c) magnetic intensity of $x=0.2$ at $1.4 \mathrm{~K}$ and $P=3.7 \mathrm{GPa}$ versus scattering vector. The fit is made with the short range model (bottom line), including longer range $\mathrm{F}$ correlations (upper line). In the inset is the temperature dependence of the correlations coefficients; (d) phase diagram for $\left(\mathrm{Tb}_{1-x} \mathrm{La}_{x}\right)_{2} \mathrm{Mo}_{2} \mathrm{O}_{7}$ in the threshold region. The open symbols are measured at ambient pressure for several $x$ contents. The solid symbols are for $x=0.2$ under pressure, taking into account ESRF data to determine $a(P)$.

[22]. The first term of the function relevant for temperatures below $T_{C}$ corresponds to the depolarization by spin fluctuations perpendicular to the direction of the muon spin, whereas the second term reflects the precession of the muon spin in the average local field $\left\langle B_{\text {loc }}\right\rangle$ at the muon site. The transverse relaxation rate $\lambda_{T}$ can have both static and dynamical character. Both expressions of $P_{Z}(t)$ are expected to merge in the high temperature limit, when the dynamics of $\mathrm{Tb}^{3+}$ and $\mathrm{Mo}^{4+}$ moments is fast, yielding $\lambda_{Z}=\lambda_{T},\left\langle B_{\text {loc }}\right\rangle=0$, and $\beta=1$. We notice that a fit with a dynamical Kubo-Toyabe function is possible for a small temperature range below $T_{C}$, but the function used above gives better results at low temperature and close to $T_{C}$. The background contribution from the cryostat walls was determined at $70 \mathrm{~K}$ in a transverse field of $50 \mathrm{G}$. The total function is written as $P_{Z}(t)=0.9 P_{Z}(t)+0.1 \exp (-0.1 t)$. Spectra are shown in Fig. 4(a).

The longitudinal relaxation rate $\lambda_{Z}$ shows a cusp at $T_{C} \sim$ $60 \mathrm{~K}$, then a broad maximum at $T^{\star} \sim 25 \mathrm{~K}$. The transverse relaxation rate $\lambda_{T}$ is about 10 times larger than $\lambda_{Z}(T)$. It smoothly increases below $T_{C}$ in a way similar to the average local field $\left\langle B_{\text {loc }}\right\rangle$. This leads us to assign $\lambda_{T}$ mainly to the width of the distribution of local fields. Both quantities scale with the ordered moment $M_{\mathrm{Tb}}(T)$ measured by 

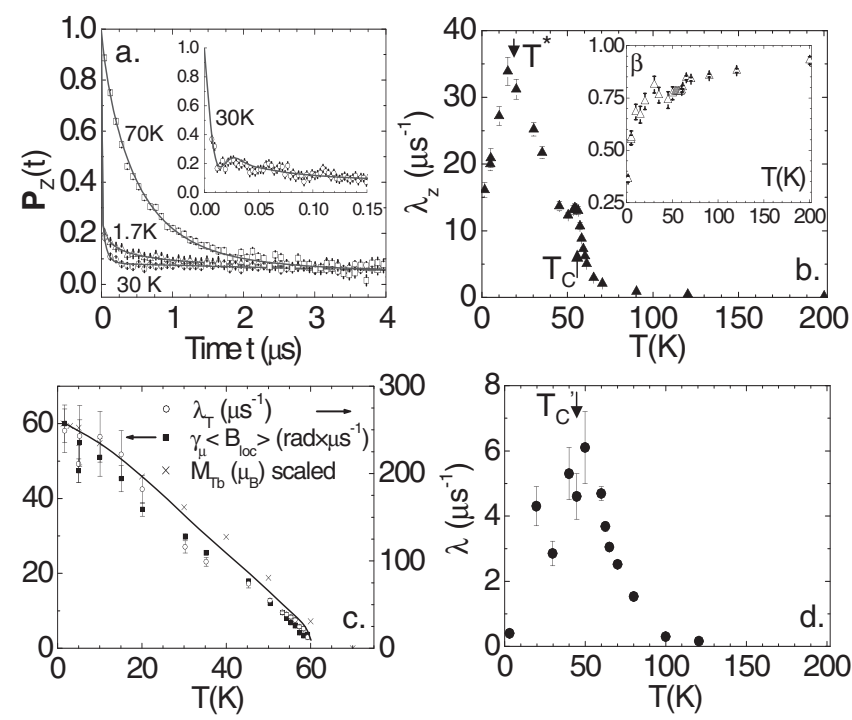

FIG. 4. $\mu \mathrm{SR}$ results for $x=0.2$. (a) Muon depolarization function $P_{Z}(t)$ at ambient pressure for several temperatures, taking into account the background term. (b) Temperature dependence of $\lambda_{Z}$ and $\beta$ at ambient pressure; (c) $\left\langle B_{\text {loc }}\right\rangle, \lambda_{T}$, and $M_{\mathrm{Tb}}$ (scaled) at ambient pressure; (d) $\lambda$ at $P=1.3 \mathrm{GPa}$.

neutron diffraction. It suggests that the local field seen by the muon comes mostly from the $\mathrm{Tb}^{3+}$ ions with much larger moments, although more localized, than the $\mathrm{Mo}^{4+}$ ones. Previous $\mu$ SR data [7] also show that the static internal field is about 10 times larger in $\mathrm{Tb}_{2} \mathrm{Mo}_{2} \mathrm{O}_{7}$ than in $\mathrm{Tb}_{2} \mathrm{Mo}_{2} \mathrm{O}_{7}$ spin glass.

Under pressure and below $T_{C}$, it was difficult to extract any information from the $\mu$ SR spectra at small times (the $2 / 3$ term), due to the large background of the pressure cell and the fast depolarization of the $2 / 3$ term. Therefore, below $T_{C}$ we fitted the data only to the $1 / 3$ term, with an exponential depolarization function, skipping the first $0.2 \mu \mathrm{s}$ of the $\mu \mathrm{SR}$ spectra. The $T_{C}$ value decreases under pressure, in agreement with the neutron data, and the anomaly at $T^{\star}$ is strongly suppressed. The results at $1.3 \mathrm{GPa}$ suggest that $T_{C}$ will tend to merge with $T^{\star}$ as pressure increases further, so that a single spin-glass transition will occur.

The anomaly at $T^{\star}$ is akin to that observed by $\mu \mathrm{SR}$ in $\mathrm{Sm}_{2} \mathrm{Mo}_{2} \mathrm{O}_{7}$ [23]. It also recalls observations [24,25] in reentrant spin glasses (RSG's). In contrast, it does not seem to exist in $\mathrm{Gd}_{2} \mathrm{Mo}_{2} \mathrm{O}_{7}$ with the isotropic $\mathrm{Gd}$ ion, where the ground state is a collinear ferromagnet [21]. This shows that, in $R_{2} \mathrm{Mo}_{2} \mathrm{O}_{7}$ compounds, the occurrence of the $T^{\star}$ anomaly does not require chemical disorder like in RSG's. It may be induced by the crystal field anisotropy of the $\mathrm{R}$ ion, either uniaxial ( $\mathrm{Tb}$ and $\mathrm{Nd}$ ) or planar $(\mathrm{Sm})$, and the consecutive frustration of the ferromagnetic state.
We notice that the long range magnetic order does not break at $T^{\star}$ and that there is no anomaly in the ordered moments or canting angles. The static field seen by the muon shows no anomaly either. It suggests a dynamical effect, possibly connected with the freezing of the short range correlated moments. We could check it in the future by inelastic neutron scattering.

In conclusion, in $\left(\mathrm{Tb}_{1-x} \mathrm{La}_{x}\right)_{2} \mathrm{Mo}_{2} \mathrm{O}_{7}$ we showed that long range magnetic order can be induced by diluting the $\mathrm{Tb}$ lattice with a nonmagnetic La ion and then be destroyed under pressure. We could study the microscopic changes of magnetism when crossing the F-SG threshold, for both statics and dynamics.

We thank A. Amato and U. Zimmermann for $\mu \mathrm{SR}$ measurements on GPS and GPD (PSI), W. Crichton for X-ray measurements on ID27 (ESRF), F. Bourée and G. André for neutron measurements on 3T2 and G41 (LLB), and A. Forget and D. Colson for the sample preparation (SPEC).

[1] S. T. Bramwell and M. J.P. Gingras, Science 294, 1495 (2001).

[2] J. E. Greedan, J. Mater. Chem. 11, 37 (2001).

[3] T. Katsufuji et al., Phys. Rev. Lett. 84, 1998 (2000).

[4] Y. Moritomo et al., Phys. Rev. B 63, 144425 (2001).

[5] J. S. Kang et al., Phys. Rev. B 65, 224422 (2002).

[6] I. V. Solovyev, Phys. Rev. B 67, 174406 (2003).

[7] S. R. Dunsiger et al., Phys. Rev. B 54, 9019 (1996).

[8] J.E. Greedan et al., Phys. Rev. B 43, 5682 (1991).

[9] B. D. Gaulin et al., Phys. Rev. Lett. 69, 3244 (1992).

[10] J. S. Gardner et al., Phys. Rev. Lett. 83, 211 (1999).

[11] Y. Yasui et al., J. Phys. Soc. Jpn. 70, 284 (2001).

[12] Y. Taguchi et al., Science 291, 2573 (2001).

[13] H. C. Kim et al., Acta Phys. Pol. B 34, 1429 (2003); Physica (Amsterdam) 359B, 1246 (2005).

[14] J.-G. Park et al., Physica (Amsterdam) 328B, 90 (2003).

[15] J. Rodríguez-Carvajal, Physica (Amsterdam) 192B, 55 (1993).

[16] I. Goncharenko, High Press. Res. 24, 193 (2004).

[17] J. Rodríguez-Carvajal, BASIREPS, ftp://ftp.cea.fr/pub/llb/ divers/BasIreps.

[18] Y. A. Izyumov et al., Neutron Diffraction on Magnetic Materials (Consultants Bureau, New York, 1991).

[19] M. Sato and J.E. Greedan, J. Solid State Chem. 67, 248 (1987).

[20] I. Mirebeau et al., Phys. Rev. Lett. 94, 246402 (2005).

[21] I. Mirebeau et al., cond-mat/0606420.

[22] P. Dalmas de Réotier, P. C. M. Gubbens, and A. Yaouanc, J. Phys. Condens. Matter 16, S4687 (2004).

[23] Y. Jo et al., J. Korean Phys. Soc. 47, 123 (2005).

[24] I. Mirebeau et al., Hyperfine Interact. 104, 343 (1997).

[25] D. H. Ryan et al., Phys. Rev. B 61, 6816 (2000). 